\title{
ON THE ABSORPTION OF INFRARED RADIATION BY ELECTRONS IN STEP QUANTUM WELLS*
}

\author{
M. Zalużny and C. Nalewajko \\ Department of Theoretical Physics, Maria Curie-Skłodowska University \\ pl. M. Curie-Skłodowskiej 1, 20-031 Lublin, Poland
}

The influence of the depolarization effect on the spectral characteristics of intraband absorption on bound-free transitions in semiconductor quantum wells is analyzed using the local and nonlocal theories. The results obtained show that the local theory only qualitatively describes the modification of the bound-free absorption spectrum by the depolarization effect.

PACS numbers: 78.30.Fs, 73.20.Dx

\section{Introduction}

It is well known that the depolarization effect (DE) must be taken into account when an electron is excited from the localized state in the quantum well (QW) systems. The DE was first discovered by Chen et al. [1]. In their model the quasi-two-dimensional electron gas was represented by an anisotropic plasma slab (with the effective thickness $d_{\text {eff }}$ ) whose spatially uniform conductivity tensor is the average conductivity of the original nonlocal system. (Note that $d_{\mathrm{eff}}$ is an adjustable parameter in this local model.)

A more sophisticated nonlocal (NL) theory was developed by Allen et al. [2]. Unfortunately, the NL theory is much more complicated when the several subbands are involved in the transitions. We have such a situation in the case when the energy of the second subband $E_{2}$ is nearly at the top of the barrier (or when the intersubband transitions final states are above the barrier). For this reason in all papers $[4,5]$ discussing the influence of the $\mathrm{DE}$ on the bound-to-continuum transitions in QW systems the local approximation (LA) developed in Ref. [1] was used. At this point it is worth to note that the problem of influence of the DE on the bound-to-continuum transitions is interesting because the performance of the quantum well infrared detectors dramatically improves when electrons are excited from the bound states in the well to the extended continuum states [7].

*This work is supported under grant No. 2P30219106 of the State Committe for Scientific Research (Republic of Poland). 


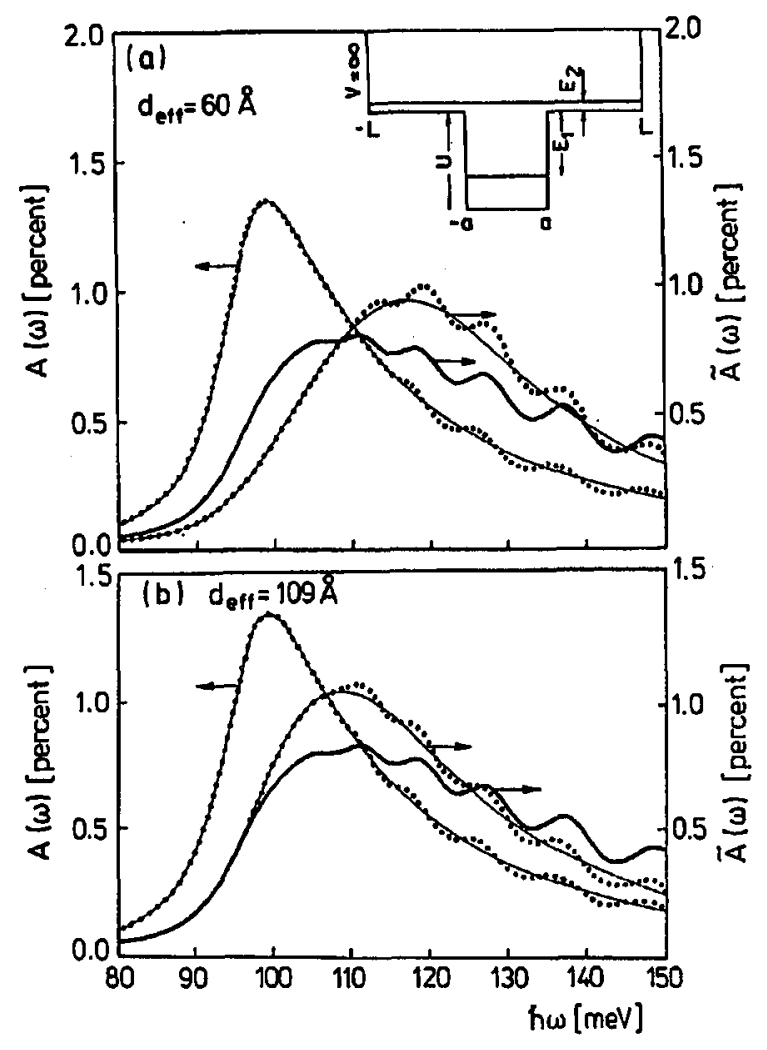

Fig. 1. Absorption $\tilde{A}(\omega)$ and $A(\omega)$ as a function of photon energy $\hbar \omega$ for the GaAs QW with $a=30 \AA, L=900 \AA, U=150 \AA, N_{s}=10^{12} \mathrm{~cm}^{-2}, \Gamma=10 \mathrm{meV}$, calculated for two values of $d_{\text {eff }}$ : (a) $d_{\text {eff }}=2 a$ and (b) $d_{\text {eff }}=2\left(a+\lambda_{0}\right)$. (....) LA model, (thick line) NL model, (thin line) LA model with $L \rightarrow \infty$.

The purpose of this paper is to estimate how reliable the LA can be in the above-mentioned situation. We will compare the absorption spectra obtained in the local and nonlocal theories for the step quantum well system (see inset of Fig. 1) assuming that the bound states are localized in the narrow QW of a width $2 a$. The excited states (localized in the well of a width $2 L \gg 2 a$ and infinite potential at both ends) can be treated (in the first approximation) as above barrier continuum states. More realistic situation $(L \rightarrow \infty)$ will be considered in a separate paper.

\section{Theory}

Due to the depolarization effect, the electronic motion in the $z$ direction normal to the interface is better described by the modified 2D conductivity $\tilde{\sigma}_{z z}$ (current-displacement-field response function) rather than by the usual 2D conductivity $\sigma_{z z}$ (current-electric field response function). Consequently, in the case of the bound-bound $(b-b)$ and bound-free $(b-f)$ transitions, induced by IR light 
polarized in the $z$-direction, the absorption of a single QW takes the form $[4,6]$

$$
\tilde{A}(\omega)=(4 \pi / c n) \operatorname{Re} \tilde{\sigma}_{z z}(\omega),
$$

where $n=\epsilon^{1 / 2}, \omega$ is the frequency of IR, $\epsilon$ is the dielectric constant of the constituent materials and is taken to be 11 for our calculations (GaAs).

In the LA $\tilde{\sigma}_{z z}(\omega)$ is given by the formula [1]

$$
\tilde{\sigma}_{z z}(\omega)=\sigma_{z z}(\omega)\left[1+\mathrm{i} \gamma(\omega) \sigma_{z z}(\omega)\right]^{-1},
$$

where $\gamma(\omega)=4 \pi / \epsilon \omega d_{\text {eff }}$ and $\sigma_{z z}(\omega)$ is the $z z$ component of the complex two-dimensional conductivity tensor given by

$$
\sigma_{z z}(\omega)=\frac{-\mathrm{i} N_{s} e^{2} \omega}{m} \sum_{f} \frac{f_{f}}{\left(\omega_{f}^{2}-\omega^{2}\right)-\mathrm{i} \Gamma \omega / \hbar} .
$$

$\Gamma$ is the phenomenological parameter describing the line broadening induced by the electron scattering, $\omega_{f}=\left(E_{f}-E_{1}\right) / \hbar, m$ is the effective mass, $f_{f}$ is the oscillator strength corresponding to $|1\rangle \rightarrow|f\rangle$ transitions and $N_{s}$ is the electron concentration in a unit area. (We assume that only ground state $n=1$ is occupied.)

In the NL case we have

$$
\tilde{\sigma}_{z z}(\omega)=\frac{-\mathrm{i} N_{s} e^{2} \omega}{m} \sum_{f} \frac{\tilde{f}_{f}}{\left(\tilde{\omega}_{f}^{2}-\omega^{2}\right)-\mathrm{i} \Gamma \omega / \hbar},
$$

with $\left(\hbar \tilde{\omega}_{f}\right)^{2}=\tilde{A}_{f f}=\left(U^{-1} A U\right)_{f f}, \tilde{f}_{f}=\left[\sum_{k} f_{k}^{1 / 2} U_{k f}\right]^{2}$ and

$$
A_{k f}=\left(\hbar \omega_{f}\right)^{2}\left[\delta_{k f}+\left(\omega_{f} / \omega_{k}\right)^{1 / 2} 8 N_{s} \pi e^{2} L_{k f} / \hbar \omega_{k} \epsilon\right],
$$

where $L_{k f}$ is the Coulomb length $[2,6], U$ is the transformation matrix which we define in such a way that $\tilde{A}=U^{-1} A U$ is diagonal.

\section{Results and conclusion}

Figure 1 shows the variation of $\tilde{A}(\omega)$ and $A(\omega)$ (三 the absorption in absence of the DE) with $\hbar \omega$ for GaAs QW $\left(m=0.066 m_{0}\right)$. The calculations have been done for two values of the effective thickness: $d_{\mathrm{eff}}=2 a$ and $d_{\mathrm{eff}}=2\left(a+\lambda_{0}\right)$, where $\lambda_{0}\left(\equiv \hbar /\left(2 m E_{1}\right)^{1 / 2}\right)$ is the mean penetration depth of the electron ground state wave function.

The results obtained show that in the case of the bound-free intraband absorption the depolarization effect not only shifts the position of the absorption peak, but also enlarges its half-width and decreases its height. Unfortunately, the results predicted by the LA model are strongly dependent on $d_{\text {eff }}$ and it is not possible to obtain the coincidence between the local and nonlocal results by fitting $d_{\text {eff }}$. Thus the LA only qualitatively describes the modification of the $\mathrm{b}-\mathrm{f}$ absorption spectrum by the depolarization effect.

\section{References}

[1] W.P. Chen, Y.J. Chen, E. Burstein, Surf. Sci. 58, 263 (1976).

[2] S.J. Allen, D.C. Tsui, B. Vinter, Solid State Commun. 20, 425 (1976). 
[3] M. Załużny, Phys. Status Solidi 161, K19 (1990).

[4] M. Załużny, Solid State Commun. 79, 1013 (1991).

[5] V.V. Osipov, F.L. Serzhenko, V.D. Shadrin, Fiz. Tekh. Poluprovodn. 23, 809 (1989).

[6] T. Ando, A. Fowler, F. Stern, Rev. Mod. Phys. 54, 437 (1982).

[7] B.F. Levine, J. Appl. Phys. 74, R1 (1993). 